\title{
Changes of Extracellular Polymeric Substance (EPS) during Microcystis Aeruginosa Blooms at Different Levels of Nutrients in a Eutrophic Microcosmic Simulation Device
}

\author{
Qi Wang ${ }^{1,2 *}$, Wenjing Pang ${ }^{1}$, Yingdan $\mathrm{Mao}^{3}$, Shujie Ge${ }^{1}$, Hengguo $\mathrm{Yu}^{2}$, \\ Chuanjun Dai ${ }^{1,2}$, Min Zhao ${ }^{1,2 * *}$ \\ ${ }^{1}$ College of Life and Environmental Science, Wenzhou University, Wenzhou, China \\ ${ }^{2}$ Key Laboratory for Subtropical Ocean and Lake Environments and Biological Resources \\ Utilization Technology of Zhejiang, Wenzhou University, Wenzhou, China \\ ${ }^{3}$ Zhejiang Blue in Environmental Technology Co., Ltd., Wenzhou, China
}

Received: 1 November 2018

Accepted: 27 December 2018

\begin{abstract}
The outbreak of cyanobacterial blooms in water can cause serious harm to water supply safety and human health, which has become one of the major water environmental problems in the present world. In this paper, the representative Microcystis aeruginosa blooms were studied by the eutrophic microcosmic simulation device between natural lake system and test in laboratory scale. Toxic Microcystis aeruginosa FACHB-905 and non-toxic Microcystis aeruginosa FACHB-469 were used to simulate algal blooms in different nutrient conditions. The study of stratified EPS extracted from different vegetative and growth stages of Microcystis aeruginosa showed that high nutritional conditions can induce Microcystis aeruginosa to produce more extracellular polysaccharides, while low nutrient conditions can affect the fluorescence components in the SL-EPS (Soluble EPS) of Microcystis aeruginosa. Furthermore, the proteins in TB-EPS (Tightly bound EPS) will gradually release to SL-EPS and LB-EPS (loosely bound EPS) during the bloom, which can decrease from $34 \%$ in the initial growth period to $20 \%$. The tryptophan-like substances in SL-EPS and TB-EPS, and the humic-acid-like substances in SL-EPS have a significant impact on the Microcystis aeruginosa blooms. Finally, all these results are expected to be of use in the study of the algal blooming outbreak early warning and control problems.
\end{abstract}

Keywords: Microcystis aeruginosa, algal bloom, stratified extracellular polymeric substances (EPS), nutritional conditions

*e-mail: victor527@126.com

**e-mail: zmonzj@sina.com 


\section{Introduction}

Extracellular Polymeric Substance (EPS) secreted by microbial flora has been considered an important factor in microbial aggregation and biofilm formation $[1,2]$. Cyanobacterial blooms occurred with the production of cyanobacterial extracellular polymeric substances (EPS) [3]. Cyanobacteria EPS was a macromolecule viscous compound that binds several to hundreds of cyanobacterial cells in diameter of from 4 to $6 \mu \mathrm{m}$ amorphous algae colloidal aggregates with a diameter of up to $1 \mathrm{~cm}$ [4]. EPS was a metabolite or autolysate secreted by microorganisms, which is usually composed of proteins, polysaccharides, a small amount of nucleic acid and other substances, and can protect the microbial cells [5]. Polysaccharides and proteins account for about $70-80 \%$ of the total mass of cyanobacteria EPS, while nucleic acids, lipids, humic acid and uronic acid account for only $10-20 \%$ of the total EPS [6, 7]. It has been reported that cyanobacteria EPS is also involved in the aggregation and development of microorganisms $[8,9]$. When the external environmental conditions are suitable for the growth of cyanobacteria, the cyanobacterial colonies producing EPS in lakes will pool together and float to the surface to form cyanobacteria blooms. In addition, the formation of a "blue-green algae mat" is also considered to be due to the cyanobacteria embedded in the gel-like EPS matrix [10], its role is to further protect the blue algae from adverse environmental conditions [11]. Xu et al. studied the role of EPS in the formation of Microcystis and the formation of bloom $[12,13]$. It was found that EPS extract decreased the cohesion of Microcystis algae by increasing the energy barrier and the second confinement. Therefore, we can speculate that EPS plays a key role in the growth and development of cyanobacterial bloom.

Although we have been studying the cyanobacterial blooms, the critical factors and mechanisms of their outbreak have hitherto not been well known. At this stage, researchers from all over the world have taken various research methods to explore the formation mechanism of cyanobacterial blooms. The comprehensive methods are broadly classified into two categories: one is field investigation and research, that is, researcher use of natural lakes for field experiments, but the complexity of scene geography, climate and hydrology often make for unsatisfactory field survey research results. The other is the simulation research of the formation mechanism of the city's water bloom by using the laboratory small equipment such as a light incubator. Although the simulation method can regulate a variety of blooming conditions, such as light, temperature, nitrogen and phosphorus nutrients, its research results also apply to natural systems, but there are still many limitations. It has important environmental and ecological significance to clarify the formation mechanism of cyanobacteria blooms as the key to preventing and controlling blooms. Studying the cyanobacteria bloom process can provide a scientific basis for reducing the harm they cause. In addition, understanding the compositional characteristics of EPS in cyanobacteria; grasping the behavioral characteristics and functions of Cyanobacterial EPS during blooms; to investigate the effect of extracellular cyanobacteria on migration and transformation of other toxic and harmful substances (such as heavy metals) in water during the bloom period, and the feasibility of the "crisis cure danger," which has very important practical significance and research value for the treatment of cyanobacteria bloom and the development and even utilization of cyanobacterial EPS.

It has been reported that the growth and proliferation of Microcystis is associated with many complex environmental factors, including climatic conditions and nutrients $[14,15]$. The extracellular polymers produced during the outbreak of Microcystis bloom were thought to affect the surface stickiness of algae cells, thereby contributing to the large-scale accumulation of some algae and triggering blooms $[8,16]$. Some studies showed that the EPS of algae in the different growth stages of algae dynamic changes may play an important role in the bloom process [17]. Most of the previous investigations into Microcystis aeruginosa investigated the sampling of cyanobacteria EPS at specific time points [18], such as the exponential growth phase or the stabilization phase. However, for the entire growth stage of Microcystis aeruginosa or the dynamic changes of EPS in bloom process are still lack of characterization. In addition, before this, algae stratified EPS in the whole process of blooming outbreak of the dynamic structural effect has not yet been explored.

Therefore, in this paper, the representative Microcystis aeruginosa from the cyanobacteria of algae was selected as the research object. The simulation culture of algal blooms under different nutrient conditions was carried out using Microcystis aeruginosa toxigenic FACHB-905 and non-toxigenic FACHB-469 based on the eutrophication microuniverse simulation device between the natural lake system and the laboratory scale. In this paper, the stratified EPS of Microcystis aeruginosa bloom under different nutrition levels were extracted and analyzed in order to understand the dynamic changes of each component in the cyanobacteria-stratified EPS and its effect in the outbreak of bloom. Analysis of the response characteristics of cyanobacteria EPS to cyanobacteria blooms can help us further understand the outbreak mechanism of cyanobacterial bloom, and provide a certain support for the research on algal bloom control in lake and library.

\section{Experimental}

\section{Microcystis Aeruginosa and Culture Conditions}

Microcystis aeruginosa toxigenic FACHB-905 isolated from Lake Dianchi in Kunming in the summer 
Table 1. Culture medium Design for bloom simulation of Microcystis aeruginosa.

\begin{tabular}{|c|c|c|c|}
\hline $\begin{array}{l}\text { Cultivate } \\
\text { cylinder }\end{array}$ & BG11 (L) & $\begin{array}{l}\text { Experimental } \\
\text { water }(\mathrm{L})\end{array}$ & $\begin{array}{l}\text { Algae species } \\
(\mathrm{mL})\end{array}$ \\
\hline BG11 & 80 & 0 & \multirow{5}{*}{$\begin{array}{c}\text { FACHB-905 } \\
50 \mathrm{~mL}\end{array}$} \\
\hline 1/5 BG11 & 16 & 64 & \\
\hline 1/10 BG11 & 8 & 72 & \\
\hline 1/50 BG11 & 1.6 & 78.4 & \\
\hline 1/100 BG11 & 0.8 & 79.2 & \\
\hline BG11 & 80 & 0 & \multirow{5}{*}{$\begin{array}{c}\text { FACHB-469 } \\
50 \mathrm{~mL}\end{array}$} \\
\hline 1/5 BG11 & 16 & 64 & \\
\hline 1/10 BG11 & 8 & 72 & \\
\hline 1/50 BG11 & 1.6 & 78.4 & \\
\hline 1/100 BG11 & 0.8 & 79.2 & \\
\hline
\end{tabular}

of 1998 and non-toxigenic FACHB-469 isolated from cyanobacterial blooms in France were provided by the Institute of Hydrobiology, Chinese Academy of Sciences. Cells were cultured in BG11 medium at $25^{\circ} \mathrm{C}$ under an illumination box at an intensity of 2500 Lux with a light:dark period of 12:12 h. Before inoculation, Microcystis aeruginosa (algae density at 106 cell $/ \mathrm{mL}$ ) was centrifuged with $\mathrm{NaHCO}_{3}$ $(15 \mathrm{mg} / \mathrm{L})$ solution at $4000 \mathrm{r} / \mathrm{min}$ for $5-10 \mathrm{~min}$ and washed with sterile deionized water to remove the nutrients attached on algal cells [18]. Experimental water was collected from Wujiayuan Reservoir, which would be filtered to remove large particles of impurities, plankton and algae in water samples immediately with $0.45 \mu \mathrm{m}$ microporous membrane and returned to the laboratory. The experimental water and BG11 culture medium were prepared in different proportions (Table 1). FACHB-905 and FACHB-469 of the prepared Microcystis aeruginosa were respectively inoculated into different nutrient conditions of the medium.

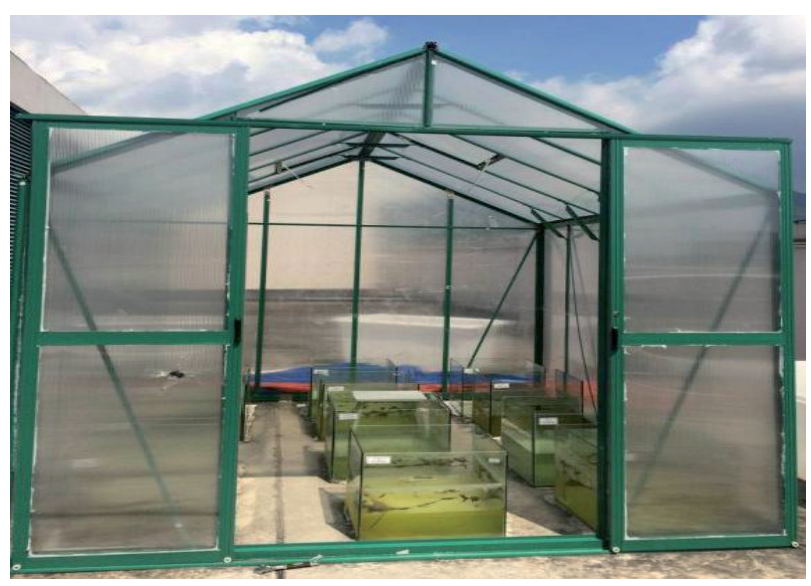

Fig. 1. Simulated culture device of Cyanobacteria bloom.
In this study, two sets of parallel micro-universe models were used to simulate the lake water bloom under eutrophication, the culture device was a transparent glass jar with $50 \times 50 \times 45 \mathrm{~cm}$ of Length $\times$ Width $\times$ Height, and the thickness of $1 \mathrm{~cm}$. The simulated eutrophic water mainly used BG11 medium as a reference, which is suitable for the growth of Microcystis aeruginosa. The simulation experiment of Microcystis aeruginosa bloom process was carried out in a rooftop sunroom (Fig. 1). Although the daily climate changed and environmental conditions were different, the temperature, light intensity, wind speed and weather were basically the same for each experimental jar in the sunroom.

\section{Sampling Program and EPS Sample Preparation}

The duration of the cultivation period was 30 days. Samples were harvested every other day during the blooms. From the beginning of the outbreak of blooms on August 3, a total of $250 \mathrm{~mL}$ samples were taken from the center of each culture cylinder at a distance of $5 \mathrm{~cm}$ from the water surface at 13:00 for the extraction and analysis of algae EPS. After every other day, water samples were taken until the death of Microcystis aeruginosa, and the end of blooms, a total of 10 times per cylinder sampling. According to the existence of EPS, the EPS is divided into dissolved state (SL-EPS) and attached state (B-EPS), B-EPS is further divided into a loosely attached state (LB) and a tightly adhered state (TB). The collected samples were extracted by centrifugation during the period of algae growth and reproduction [19], and this EPS extraction method would not cause cell lysis [20]. The collected samples were firstly centrifuged at $2500 \mathrm{~g}$ for $15 \mathrm{~min}$ at $4^{\circ} \mathrm{C}$, and the separated liquid was sucked for measurement of SL-EPS. Secondly, the harvested bloom samples were suspended in a $0.05 \% \mathrm{NaCl}$ solution and centrifuged at $4500 \mathrm{~g}$ for $15 \mathrm{~min}$ at $4^{\circ} \mathrm{C}$, with the liquid collected carefully for measurement of LB-EPS. Thirdly, the remaining bloom samples were re-suspended with $0.05 \% \mathrm{NaCl}$ solution and treated for TB-EPS extraction using the following method. $1 \mathrm{M}$ of $\mathrm{NaOH}$ to adjusts the $\mathrm{pH}$ to be 11.0. Stir for $10 \mathrm{~min}$ with the rotation speed of $80 \mathrm{rpm}$ at $4^{\circ} \mathrm{C}$, then heat at $60^{\circ} \mathrm{C}$ for $30 \mathrm{~min}$ [21]. After extraction, all samples were centrifuged at $15000 \mathrm{~g}$ for $20 \mathrm{~min}$ at $4^{\circ} \mathrm{C}$, with the separated liquid collected carefully for measurement of TB-EPS. Finally, all the extracted solutions were filtered out through the $0.45 \mu \mathrm{m}$ PTFE membranes and stored in a brown volumetric flask at $4^{\circ} \mathrm{C}$ for further analysis.

Fluorescence EEM Determination and Determination of Organic Components

Fluorescence EEMs were measured using a fluorescence spectrometer (F-4600, Hitachi, Japan) in scan mode with a 700-voltagexenon lamp. EEM spectra were gathered with scanning emission (Em) 
spectra from $250 \mathrm{~nm}$ to $600 \mathrm{~nm}$ at $2 \mathrm{~nm}$ increments by varying the excitation (Ex) wavelength from $200 \mathrm{~nm}$ to $500 \mathrm{~nm}$ at $5 \mathrm{~nm}$ increments. The spectra were recorded at a scan rate of $1200 \mathrm{~nm} / \mathrm{min}$, using Ex and Em slit bandwidths of $5 \mathrm{~nm}$. Reaction temperature control system was high-precision water bath control system, and the control temperature was $25 \pm 1^{\circ} \mathrm{C}$. The blank scans were performed using Milli-Q water. The protein concentration was determined by BCA kit (Shanghai Sangon Biotech, C503061), and the polysaccharide concentration was determined by anthrone-sulphuric acid colrimetry.

\section{Results and Discussion}

\section{Growth of Microcystis Aeruginosa FACHB-905 and FACHB-469 under Different Nutritional Conditions}

The growth of Microcystis aeruginosa follows the general rule of microbial, that is, it undergoes four processes, including lag phase, logarithmic growth phase, stable phase and decay phase, which is one growth cycle of algae. However, the growth curve of Microcystis aeruginosa is only lag phase, logarithmic growth phase and decay phase without an obvious stable phase [22]. Some studies have shown that Cyanobacteria density is highly correlated with chlorophyll-a content [23], so the chlorophyll-a concentration will be used as a characterization of main growth indicators about the Microcystis aeruginosa bloom process. It can be seen from the growth performance of Microcystis aeruginosa toxigenic FACHB-905 and non-toxigenic FACHB-469
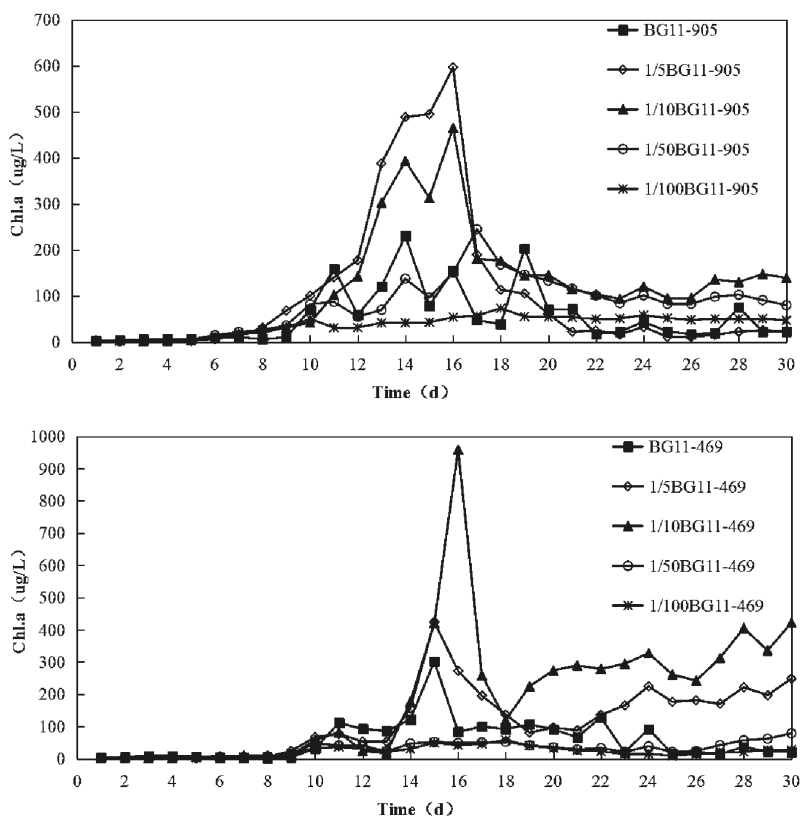

Fig. 2. Changes of Chlorophyll-a of Microcystis aeruginosa FACHB-905 and FACHB-469 under different nutrition levels. under different nutrient levels that both the FHCB-905 and FACHB-469 of the Microcystis aeruginosa contain lag phase, log phase and decay phase (Fig. 2). However, FACHB-905 could also see a stationary phase after the decline stage, and FACHB-469 also had a relapse phase at high concentrations due to the differences in the strains of Microcystis aeruginosa and the different nutrient concentrations.

Microcystis aeruginosa grows slowly when the chlorophyll-a content has basically no change in the initial stage of culture (about one week), this stage can be considered as lag phase. Some studies have suggested that the growth rate of Microcystis mainly depends on the intracellular phosphorus [24], not extracellular phosphorus, which can be confirmed from the lag phase. Microcystis aeruginosa did not quickly enter the logarithmic phase despite the high phosphorus content of water from phosphorus-deficient state that has come into the phosphorus-rich medium, which can indicate that Microcystis was quickly uptaking the extracellular phosphorus in this phase in preparation for its massive proliferation.

Microcystis aeruginosa toxigenic FACHB-905 and non-toxigenic FACHB-469 cultured under different nutrient levels entered the logarithmic growth phase after the adaptation stage. There was a slight difference in the time between the two algae in logarithmic growth phase, FACHB-905 entered logarithmic growth phase 2 to 3 days earlier than FACHB-469, which can indicate that the adaptability of the toxigenic FACHB-905 strains is stronger than non-toxigenic FACHB-469. The different levels of nutrition did not significantly affect the time of two algae populations entering the logarithmic growth phase.

The concentration of chlorophyll-a of FACHB-905 and FACHB-469 increased significantly after they entered to the logarithmic growth phase, and even the highest concentration can grow up to hundreds of micrograms per liter. Microcystis aeruginosa began to enter the decay stage from the $15^{\text {th }}$ to the $17^{\text {th }}$ day under different nutrient levels, and the chlorophyll-a concentration decreased rapidly. The non-toxigenic FACHB-469 entered the decay phase 1 to 2 days earlier than the toxigenic FACHB-905 under moderate nutrient levels (1/5 BG11 and 1/10 BG11) (Fig. 2). The chlorophyll-a concentration of FACHB-905 maintained at a steady level under different nutrient levels after the decay period ended, but the concentration was significantly higher than the original level. While non-toxigenic FACHB-469 entered the relapse period under 1/5 BG11 and 1/10 BG11 nutrition conditions, chlorophyll-a concentration began to increase again.

The changes of $\mathrm{N}$ and $\mathrm{P}$ concentrations have a significant impact on the biomass of Microcystis aeruginosa. The biomass of Microcystis aeruginosa increased with the increase of $\mathrm{N}$ and $\mathrm{P}$ concentrations when the concentrations of $\mathrm{N}$ and $\mathrm{P}$ were low. The biomass of Microcystis aeruginosa declined with the increase of $\mathrm{N}$ and $\mathrm{P}$ concentrations at higher 
concentrations. So it is not hard to understand that the biomass of Microcystis aeruginosa at the highest nutrient level is not the highest. FACHB-905 grew best under the 1/5 BG11 nutrient conditions, while FACHB-469 grew best under the 1/10 BG11 nutrient condition (Fig. 2). In addition, it was also found that FACHB-905 could not grow well under 1/100 BG11 nutrient conditions, while FACHB-469 could not grow well under 1/50 BG11 and 1/100 BG11 nutrient conditions. These results mainly indicate that FACHB-905 was more adaptable to low nutrient conditions than FACHB-469. Many researchers found that cyanobacteria cultured in a laboratory light incubator was very difficult to appear as the outbreak of bloom, but in natural conditions it was very difficult to fully study the blue algae bloom from the entire outbreak process of "start-outbreak-end." Therefore, this simulation training experiment can intuitively observe the whole process of Microcystis aeruginosa bloom outbreak, and has an important guiding significance for further understanding the outbreak mechanism of cyanobacteria bloom.

In order to more intuitively understand the organoleptic characteristics of the water during the whole process of Microcystis aeruginosa bloom, the changes of water in the culture jar were taken daily during the experiment. Microcystis aeruginosa toxigenic FACHB-905, which is cultured at 1/5 BG11 nutrition level with the most obvious growth condition, is taken as an example to observe the changes of transparency, color, odor and a series of sensory indices during the growth of Microcystis aeruginosa, and to understand the process of blooming in the most intuitive way. Fig. 3 shows a photo of the bloom process.

From the photos we can see, from the beginning of the experiment until August 2 (the eighth day of culture), that there was no significant change in the water in the culture tank, which was kept in a colorless and transparent state during the inoculation, which was the lag phase of Microcystis aeruginosa FACHB-905. Beginning August 3, we can see that the water inside the culture tank begins to turn green and the transparency decreases. August 5 to August 8, bubbles on the surface of the water, the water began to become turbid and viscous, and a stench was produced. From August 9-11, the surface of the water was covered with a blue-green algae foam, the surface was covered with a layer of oil film, and small groups of Microcystis aeruginosa were scattered on the surface. On August 12, the surface foam decreased and the surface could see a larger piece of Microcystis aeruginosa, with cyanobacteria attached to the cylinder wall and bottom. On August 13 bubbles disappeared, we could see large blue-green algae distribution in the surface and cylinder wall, and the water was turbid and thick, emitting a stench. August 14-15, large blue-green algae float on the surface and the cylinder wall, cylinder wall and bottom of the cylinder with cyanobacteria hanging film, the water began to clear, and transparency increased. This stage was the logarithmic growth phase of FACHB-905, and was the most obvious bloom process of Microcystis aeruginosa in this simulation experiment. August 16, a large number of film-like clusters of algae gathered in
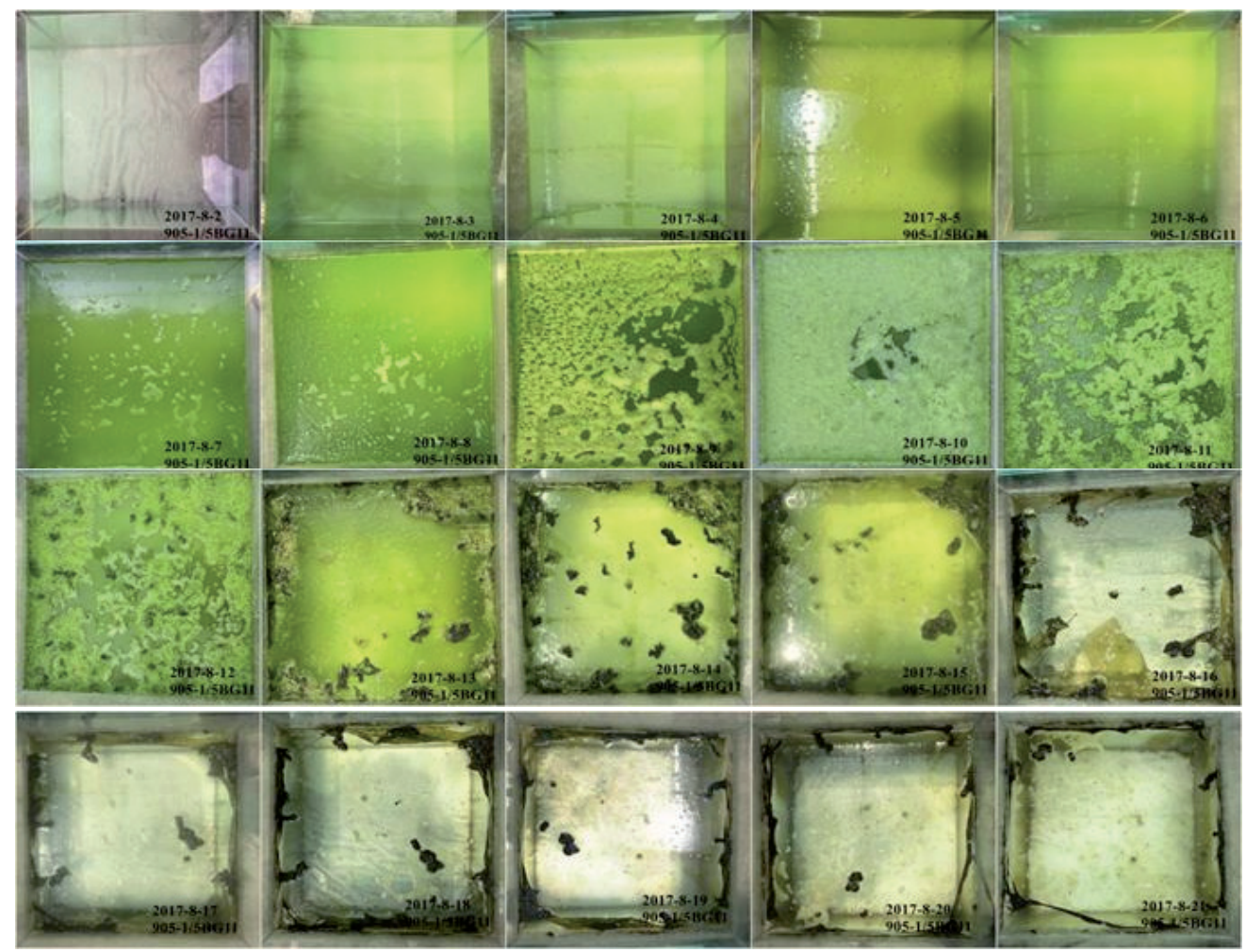

Fig. 3. Blooms of Microcystis aeruginosa FACHB-905 under best nutrient level. 
the cylinder wall around, water quality became clear. August 17 to the end of experiment, the algae began to hang on the cylinder wall and sink into the bottom of the cylinder, the color of algae become from blue-green to dark brown, the water yellowish but transparent. This stage was the decline phase of Microcystis aeruginosa FACHB-905.

In the simulation experiment we found that there was no significant difference between the Microcystis aeruginosa toxigenic FACHB-905 and non-toxigenic FACHB-469 in the whole process of blooming. However, Microcystis blooms did not appear in similar photos from August 9 to August 15 at low nutrient levels (1/50 BG11 and 1/100 BG11), but dead Microcystis aeruginosa can be seen in the cylinder after slightly yellowish greenish water.

\section{Changes of Protein Content in EPS of Microcystis Aeruginosa under Different Nutrition Conditions}

From the changes of protein content in layered EPS of Microcystis aeruginosa FACHB-905 and FACHB-469 during the entire bloom period under different nutrient levels (Fig. 4), we can see that the total protein content of EPS in Microcystis aeruginosa FACHB-905 and FACHB-469 cultured in pure BG11 medium has always been increasing. From the initial to the final extraction, the total amount of protein roughly doubled and did not show the trend of increasing first and then decreasing with the growth of algae. This is probably because the nutrient-rich pure BG11 medium can always induce the production of Microcystis aeruginosa EPS. In addition, the protein content in stratified EPS continues to increase, but it is clear that the proportion of protein content in TB-EPS continues to decline throughout the EPS. During the logarithmic growth of algae, the proteins in EPS were uniformly distributed in the layers of SL-EPS, LB-EPS and TB-EPS, and the protein content of TB-EPS decreased significantly in the total EPS, from $34 \%$ of the growth period to about $20 \%$ during the decay period. This phenomenon may due to the gradual release of proteins in the outer layer of TB-EPS during the decay period due to the death of the algae cells.

The total EPS of FACHB-905 and FACHB-469 in the whole process of algal bloom increased first (logarithmic growth phase) and then decreased (decline phase) at $1 / 5$ and 1/10 BG11 culture conditions, and FACHB-469 also shows an increase in protein content during the relapse phase. It was obvious that during the outbreak of the bloom, not only the proportion of protein but also the contents in TB-EPS decreased continuously under these two nutrient conditions. Microcystis aeruginosa FACHB-905 downed from the initial $5.899 \mathrm{mg} / \mathrm{L}$ to $2.52 \mathrm{mg} / \mathrm{L}$; FACHB-469 downed from the initial $5.019 \mathrm{mg} / \mathrm{L}$ to $2.126 \mathrm{mg} / \mathrm{L}$, and began to experience a relapsing phase increased to $3.006 \mathrm{mg} / \mathrm{L}$, that is to say, the content of protein in TB-EPS is reduced by about half from the growth stage to the decay stage.
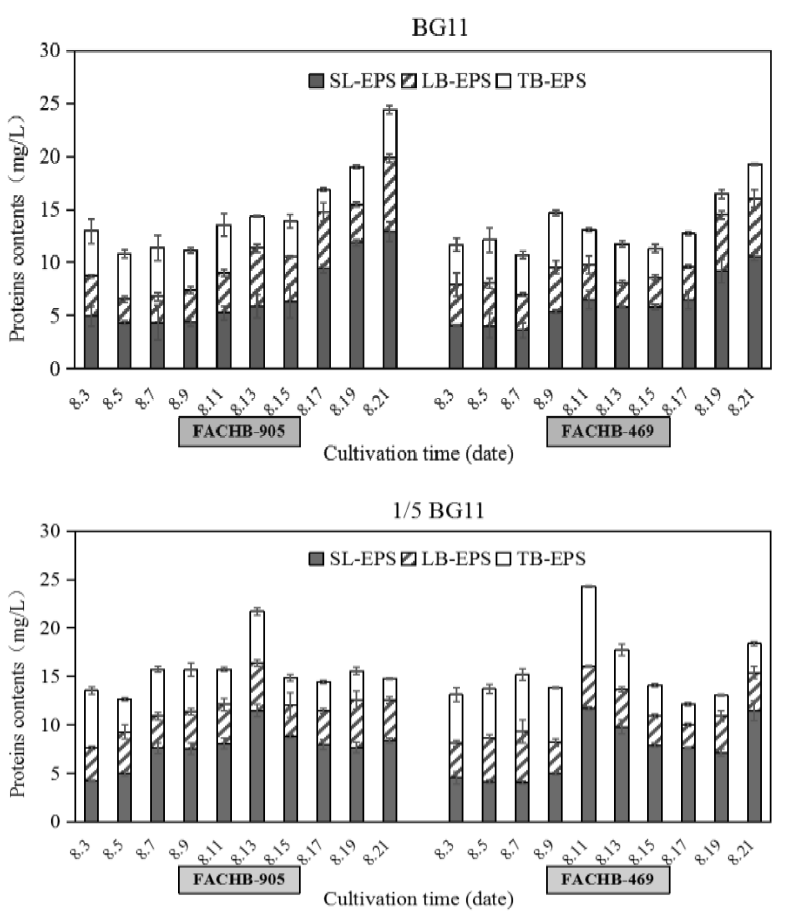

$1 / 10 \mathrm{BG} 11$

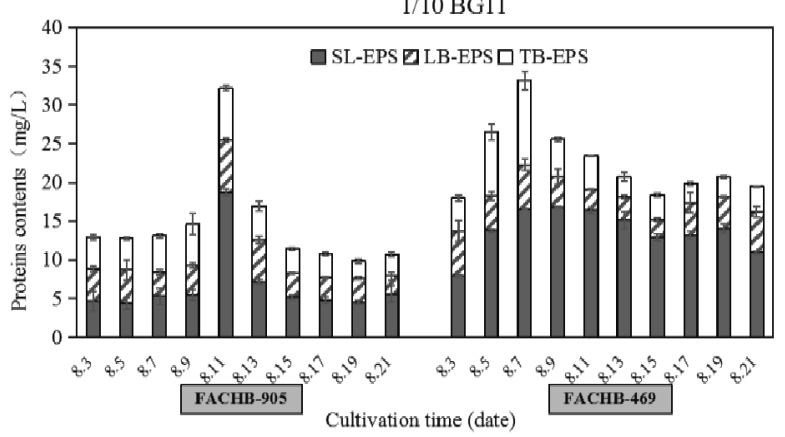

1/50 BG11

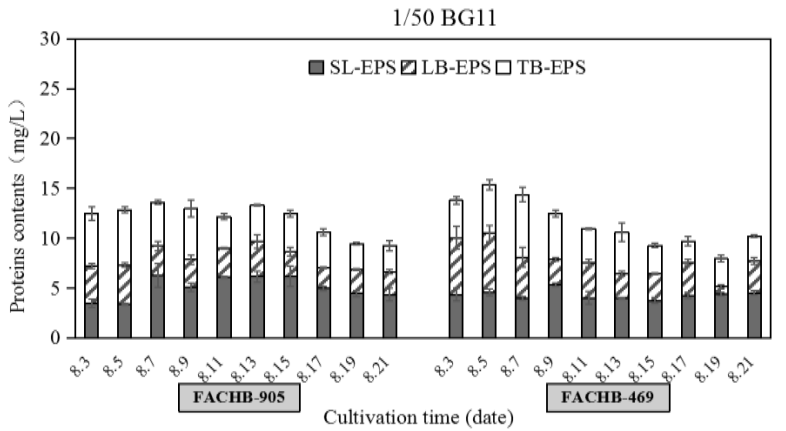

$1 / 100$ BG11

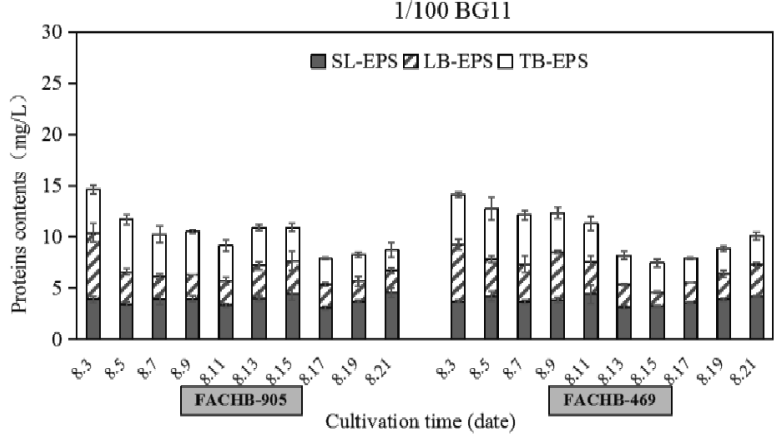

Fig. 4. Changes of Proteins in stratified EPS about Microcystis aeruginosa FACHB-905 and FACHB-469 under different nutrition levels. 
This phenomenon may be attributed to the gradual release of proteins in the outer layer of TB-EPS into SL-EPS and LB-EPS during the decay period due to the death of the algae cells. In addition, it can be seen that FACHB-905 produced by Microcystis aeruginosa cultured in 1/5 BG11 had a higher content of polysaccharide in EPS than $1 / 10$ BG11, while FACHB-469 of M. aeruginosa was the opposite, but the algae growth was consistent. On the other hand, at $1 / 50$ and 1/100 BG11 culture conditions, Microcystis aeruginosa was found to have a significantly lower protein content in EPS at later growth stages than in eutrophication (1/5 and 1/10 BG11) content.

In this study, we can see that Microcystis aeruginosa can induce more EPS production during logarithmic growth under natural simulated culture conditions. This shows that Microcystis aeruginosa can regulate its metabolism according to the growth conditions, and the metabolism is more vigorous in the logarithmic phase. Similarly, it was previously found that dissolved organic carbon in algae cells is the most abundant in the stable growth phase [17].

\section{The Changes of Polysaccharides Content in EPS of Microcystis Aeruginosa under Different Nutrition Conditions}

According to the changes of polysaccharide content in layered EPS of Microcystis aeruginosa FACHB-905 and FACHB-469 during the entire bloom period under different nutrient levels (Fig. 5), we can see: the content of total polysaccharides in the FACHB-905 and FACHB-469 showing an increasing (logarithmic growth phase) and a decreasing (declining phase) in the whole bloom process under different nutrition conditions, and the growth of Microcystis aeruginosa FACHB-469 can also be seen as increased relapsing phase in pure BG11 and 1/5 BG11 culture conditions, which showed the same trend of consistency with the growth of algae. The increase of total polysaccharide content in EPS was mainly dominated by SL-EPS, but there was a slight increase of polysaccharide content in LB-EPS during the whole growth stage, while the content of polysaccharide in TB-EPS showed no obvious change.

The content of polysaccharides in Microcystis aeruginosa EPS is different from that of protein (Fig. 5), and the content of polysaccharides remains at the level of SL-EPS $>$ LB-EPS $>$ TB-EPS, indicating that polysaccharides mainly exist in the outermost layer of Microcystis aeruginosa EPS. On the other hand, it was also found that the content of polysaccharides in FACHB-905 and FACHB-469 layered EPS was similar at low nutrient levels, with the total content maintained at about $10-15 \mathrm{mg} / \mathrm{L}$, while under high nutritional level, EPS polysaccharide content up to about $100 \mathrm{mg} / \mathrm{L}$, an increase of nearly 1.5 times the initial state, indicated that high nutritional conditions can induce Microcystis aeruginosa to produce more EPS because the carbon fixation efficiency of microcystis was
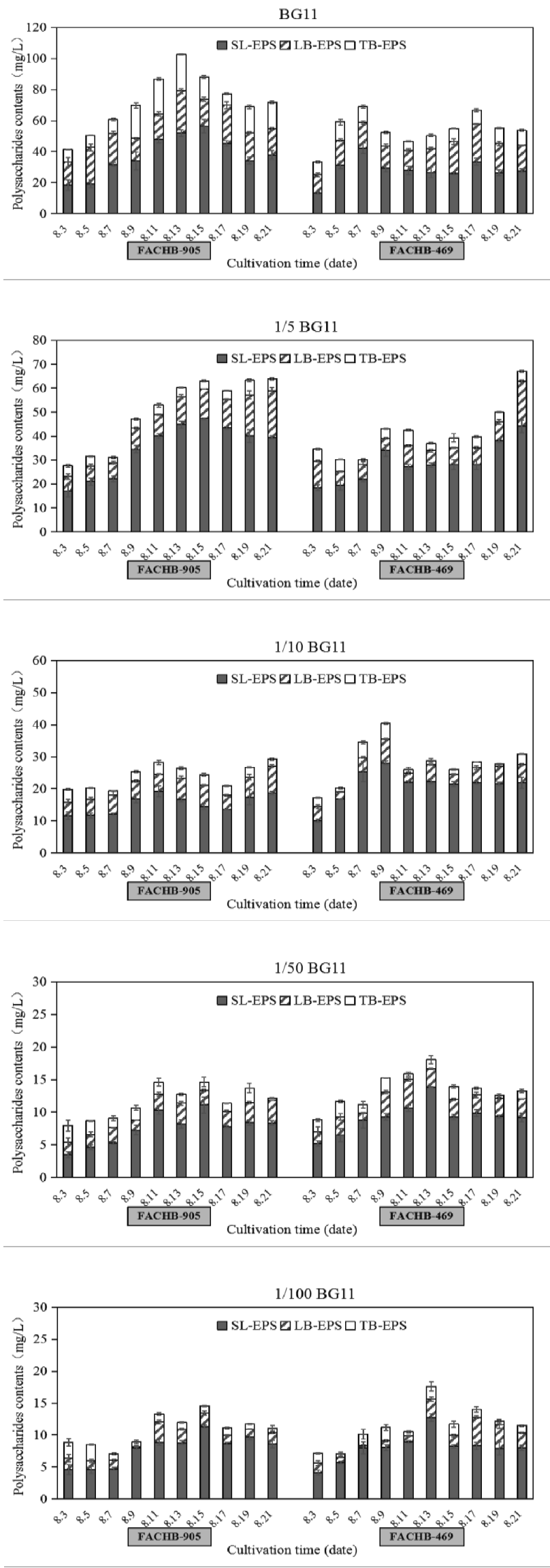

Fig. 5. Changes of Polysaccharides in stratified EPS about Microcystis aeruginosa FACHB-905 and FACHB-469 under different nutrition levels. 
high under the condition of rich nitrogen, which resulted in a large amount of synthesis of polysaccharides.

Comparing the relationship between polysaccharides and proteins, we can find that the content of polysaccharides in SL-EPS was always higher than that in protein regardless of the growth stage and nutrition level of algae, and the ratio of polysaccharides/polysaccharides among FACHB-905 and FACHB-469 were different. Microcystis aeruginosa FACHB-905 had a polysaccharide/protein ratio about 2.9-6.4 in SL-EPS, while FACHB-469 was slightly lower at 1.5-2.7; the polysaccharide/protein ratio of FACHB-905 in LB-EPS was about 1.5-4.7; and
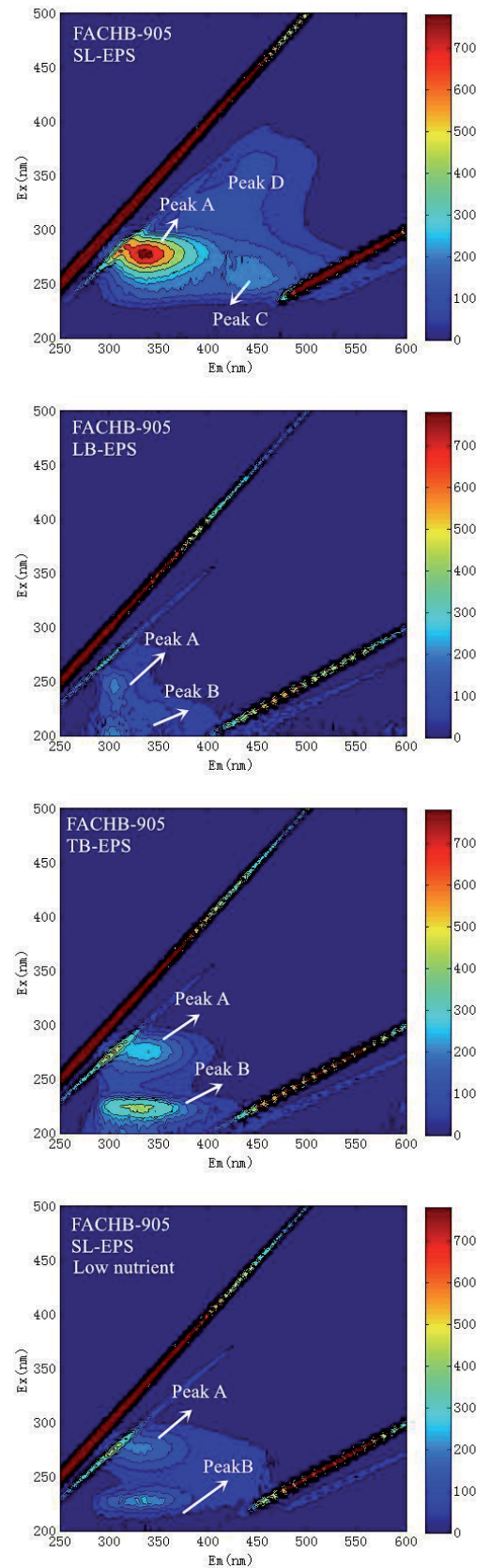

Microcystis aeruginosa FACHB-469 was about 1 . In the innermost TB-EPS, the polysaccharide/protein ratio of FACHB-905 was about 1, while the FACHB-469 was less than 1 , which indicated that the protein content is higher than polysaccharides instead. The polysaccharide/ protein ratio of FACHB-905 were all higher than FACHB-469, and the phenomenon maybe associated with toxic Microcystis aeruginosa. Qu et al. showed that polysaccharide/protein ratio of Microcystis aeruginosa EPS cultured in laboratory was about 1.25 [12]. The experimental results showed that the EPS of Microcystis aeruginosa was decreased from the outer layer to the inner layer, the ratio of polysaccharide/protein was
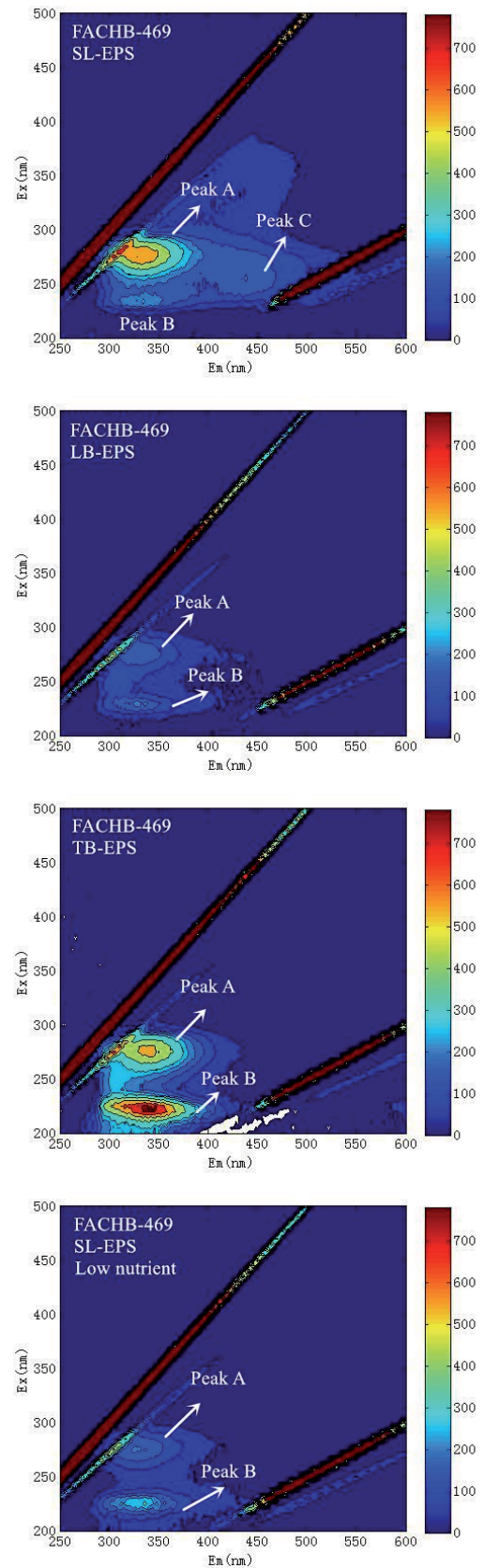

Fig. 6. Typical EEM contours of different EPS fractions under different nutritional conditions for Microcystis aeruginosa FACHB-905 and FACHB-469. 
gradually decreased, the outer layer of EPS was mainly polysaccharide and the inner layer was protein, the ratio of polysaccharide/protein in FACHB-469 was decreased more than that of FACHB-905.

The conclusion of this experiment is contrary to the conclusion of Xu's report that in the laboratory culture of Microcystis aeruginosa with BG11 medium, the content of protein and polysaccharide in SL-EPS and LB-EPS continued to decline during the logarithmic growth phase, and the decay period was significantly increased. This may be because our experiment is open outdoor simulation training experiments, closer to natural conditions, while Xu's experiments were in a closed light incubator flask conical flask; experimental conditions vary greatly.

\section{Three-Dimensional Fluorescence Characteristics of Microcystis Aeruginosa Layered EPS under Different Nutrition Conditions}

In this study, 300 three-dimensional fluorescence spectra of stratified EPS were obtained from the growth stages of Microcystis aeruginosa FACHB-905 and FACHB-469 cultured under different nutrient conditions. It can be seen from the typical three-dimensional fluorescence spectrum that
Microcystis aeruginosa FACHB-905 and FACHB-469 both present two fluorescent components in the LB-EPS and TB-EPS layers throughout the growth phase-Peak A $(E x / E m=275-280 / 340 \mathrm{~nm})$ and Peak B $(E x / E m=220-260 / 340$ nm) (Fig. 6). Both peak A and peak $\mathrm{B}$ were protein-like substances, and according to previous studies, peak $\mathrm{A}$ can be further classified as tryptophan. However, the fluorescence components of FACHB-905 and FACHB-469 in SL-EPS were different: In addition to the existence of peak A, peak B two kinds of protein components, but also appeared in the class of humic acid-like substance of the fluorescence peak $\mathrm{C}(\mathrm{Ex} / \mathrm{Em}=250 / 450 \mathrm{~nm})$ in Microcystis aeruginosa FACHB-469; FACHB-905 contains only one type of protein component of peak A, and two other types of humic acid components, namely peak $C(E x / E m=250 / 450 \mathrm{~nm})$ and peak $D$ $(\mathrm{Ex} / \mathrm{Em}=340 / 430 \mathrm{~nm})$. In the present study, the positions of peak $A$ and peak D show a certain blue shift and red shift, respectively, compared with the peak positions reported previously (proteins-like: 280-285/340-350 nm; humic acids-like: 330-340/420$430 \mathrm{~nm})$. Three-dimensional fluorescence results show that all stratified EPS are detected in the protein-like substances, while the humic acid-like substances were mainly in the SL-EPS layer.
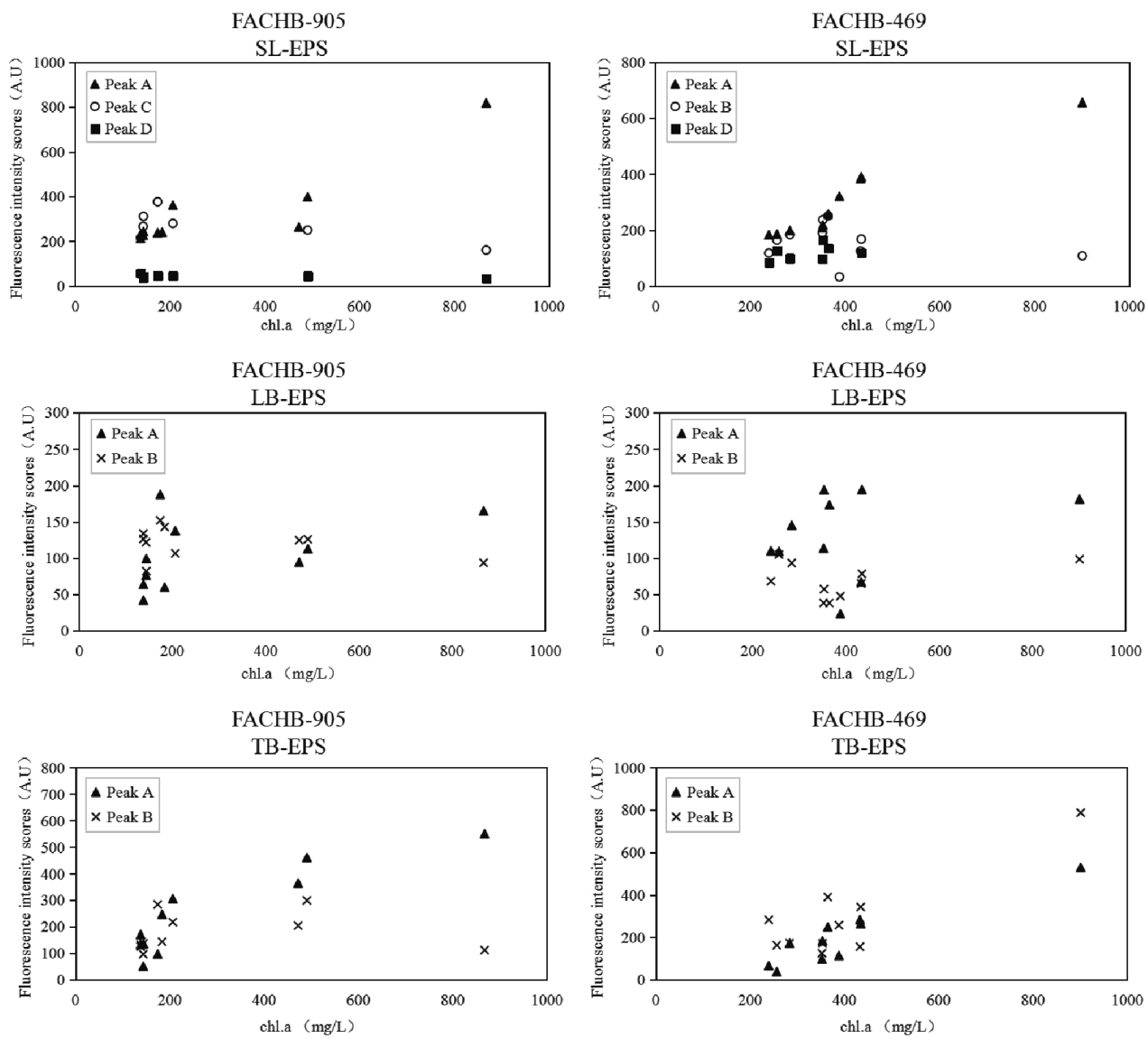

Fig. 7. The scatter plots of fluorescence intensity scores in different EPS fractions with increasing chl.a of Microcystis aeruginosa FACHB-905 and FACHB-469. 
Table 2. Pearson correlation between algae chl.a and fluorescent components of EPS produced by Microcystis aeruginosa FACHB905and FACHB-469.

\begin{tabular}{|c|c|c|c|c|c|}
\hline \multirow{2}{*}{ EPS fractions } & \multirow{2}{*}{ Components } & \multicolumn{4}{|c|}{ Microcystis aeruginosa } \\
\cline { 3 - 6 } & & $\mathrm{R}$ & \multicolumn{2}{|c|}{ FACHB-905 } & $\mathrm{R}$ \\
\cline { 3 - 6 } & Peak A & $0.901 * *$ & 0.000 & $0.767^{* *}$ & 0.010 \\
\hline \multirow{3}{*}{ SL-EPS } & Peak B/C & -0.676 & 0.096 & -0.276 & 0.441 \\
\cline { 2 - 6 } & Peak D & -0.593 & 0.160 & 0.154 & 0.741 \\
\hline \multirow{3}{*}{ LB-EPS } & Peak A & 0.462 & 0.179 & 0.286 & 0.423 \\
\cline { 2 - 6 } & Peak B & -0.344 & 0.330 & -0.16 & 0.659 \\
\hline \multirow{2}{*}{ TB-EPS } & Peak A & $0.902 * *$ & 0.000 & $0.917 * *$ & 0.000 \\
\cline { 2 - 6 } & Peak B & 0.076 & 0.834 & $0.944^{* *}$ & 0.000 \\
\hline
\end{tabular}

*Correlation is significant at the 0.05 level (2-tailed).

$* *$ Correlation is significant at the 0.01 level (2-tailed).

It is noteworthy that peaks $\mathrm{C}$ and $\mathrm{D}$ were observed in the SL-EPS extracted during the growth of Microcystis aeruginosa. Therefore, the late-growth humic acid-like fluorescence peaks were likely due to the death of algae cells and the decomposition of macromolecular organisms such as proteins and polysaccharides. In the meantime, it was also found that different nutrient conditions affected the fluorescence components of the outermost layer SL-EPS of Microcystis aeruginosa FACHB-905 and FACHB-469. Our study found that only two of the fluorescein components of SL-EPS in Microcystis aeruginosa, namely, peak $\mathrm{A}$ and peak $\mathrm{B}$, humic acid components did not appear at low nutrient levels. This result was inconsistent with the reported low nutritional level, which does not change the organic composition of EPS by Xu [15], the reason may be that the low nutrient level (1/50 or 1/100 BG11) used in this experiment was much lower than that in the $\mathrm{Xu}$ ' study (1/7 BG11).

Microcystis Aeruginosa EPS Composite Fluorescence Components and the Correlation Analysis of the Process of Water Bloom

Although fluorescence spectroscopy has long been used for the characterization of EPS, it has not yet been used to study the changes of algae EPS fluorescence components during the whole growth of Microcystis aeruginosa during bloom. According to the layout scatter of fluorescence intensity in layered EPS fluorescence peak about FACHB-905 and FACHB-469 with the chlorophyll-a in the process of bloom, and its correlation with chlorophyll-a of Microcystis aeruginosa (Fig. 7 and Table 2), here we take the measured values of Microcystis aeruginosa cultured at the optimum nutrient level as an example (FACHB-905 under 1/5 BG11 nutrient condition; FACHB-469 under 1/10 BG11 nutrient condition).
It can be seen that the fluorescence intensity of fluorescence components in Microcystis aeruginosa SL-EPS and TB-EPS was significantly higher than that in LB-EPS (Fig. 7). For SL-EPS, the fluorescence intensity of peak A was significantly higher than that of peak B (C) and peak D. At the same time, it was observed that the fluorescence intensity of peak A increased with the increase of chlorophyll-a, and the fluorescence intensity of FACHB-905 fluorescence component in SL-EPS was higher than that of FACHB-469. But no clear distribution pattern of fluorescent components was observed in LB-EPS. For TB-EPS, the fluorescence intensity of fluorescence peak A in Microcystis aeruginosa FACHB-905 was greater than that of peak B, whereas that of Microcystis aeruginosa FACHB-469 was the opposite. It can also be seen that the fluorescence intensity of peak A obviously increases with the increase of chlorophyll-a, and a similar pattern can be seen in peak B of FACHB-469, meanwhile, the fluorescence intensity of the fluorescent component in FACHB-469 is higher than FACHB-905 in this layer EPS of Microcystis aeruginosa.

It can be seen that the Pearson correlation indicates that the chlorophyll a content of FACHB-905 and FACHB-469 of Microcystis aeruginosa was significantly correlated with the partial fluorescence of EPS (Table 2). There was a significant positive correlation between chlorophyll-a content with the fluorescence intensity of peak $A(R>0.901, p<0.01)$ in SL-EPS and peak A $(\mathrm{R}>0.902, \mathrm{p}<0.01)$ in TB-EPS of Microcystis aeruginosa FACHB-905 during the blooms outbreak. These results indicated that there was a significant correlation between tryptophan species in the SL-EPS and TB-EPS of Microcystis aeruginosa FACHB-905 and the outbreak of water bloom, which can be used as the indicators of EPS fluorescence response during the outbreak of Microcystis aeruginosa bloom. The peak C and peak D of SL-EPS layer did not 
appear in the early stage, but the fluorescence intensity of these were negatively correlated with the content of chlorophyll-a during the period of algae decline after bloom, which can show that the emergence of humic substances in the SL-EPS of Microcystis aeruginosa FACHB-905 may indicate that the algae were already in decline after the outbreak of the bloom.

On the other hand, a similar situation can be observed in the EPS of Microcystis aeruginosa FACHB-469. There was a significant positive correlation between chlorophyll-a content with the fluorescence intensity of peak $A(R>0.767, p<0.01)$ in SL-EPS and peak A $(R>0.912, p<0.01)$ in TB-EPS of Microcystis aeruginosa FACHB-469 during the blooms outbreak. Moreover, the fluorescence intensity of peak B in TBEPS was also positively correlated with the growth of Microcystis aeruginosa $(\mathrm{R}>0.944, \mathrm{p}<0.01)$, which was different from that of FACHB-905.

Studies have shown that tryptophan had a high absorption and digestion effect on UV light [25]. In addition, tryptophan was also a precursor of Synthesis of UV-absorbed secondary metabolites such as scytonemin [26]. The results of three-dimensional fluorescence spectroscopy and statistical analysis showed that the tryptophan substances in SL-EPS and TB-EPS and the humic substances in SL-EPS were both associated with the FACHB-905 and FACHB-469 algae blooms.

\section{Conclusions}

FACHB-905 grew best under the conditions of 1/5 BG11, while FACHB-469 grew best under 1/10 BG11. The content of polysaccharides in Microcystis aeruginosa FACHB-905 and FACHB-469-stratified EPS always maintained at: SL-EPS $>$ LB-EPS $>$ TB-EPS at different nutrient levels and different growth stages. The polysaccharide/protein ratio decreased gradually from the outer layer to the inner layer. The high nutritional conditions can induce Microcystis aeruginosa to produce more extracellular polysaccharides, while low nutrient conditions can affect the fluorescence components in the SL-EPS of Microcystis aeruginosa. During the bloom, the content of protein and polysaccharide of Microcystis aeruginosa FACHB-905 and FACHB-469 in SL-EPS and LB-EPS continued to increase in the logarithmic growth phase, but significantly decreased in the decay phase. In the early stage of growth, the protein of Microcystis aeruginosa EPS was uniformly distributed in the EPS of SL-EPS, LB-EPS and TB-EPS; to the decay stage, the proportion of protein in TB-EPS at the total EPS significantly decreased from $34 \%$ of the growth period dropped to about $20 \%$, and the content was also continuing to decline. There were only two peak A and peak B fluorescence components in SL-EPS of Microcystis aeruginosa during the whole growing process under low nutrient level, in which the humic acid component did not appear. The tryptophan- like substances in SL-EPS and TB-EPS and humic acid-like substances in SL-EPS have a great deal of relevance with the process of Microcystis aeruginosa blooms. The aromatic amino acid tryptophan in EPS may play a major role in the bloom of Microcystis aeruginosa. The presence of tryptophan in the EPS may be an adaptation mechanism to avoid acute cellular damage and the deleterious effects of long-term UV radiation. This adaptation has great importance to the aggregation, formation and maintenance of cyanobacterial blooms on the surfaces of eutrophic freshwater systems, which deserve our further study.

\section{Acknowledgements}

This research was supported by the National Key Research and Development Program of China under grant No. 2018YFE0103700, the Zhejiang Provincial Natural Science Foundation of China under grant Nos. LY16B070008 and LQ18C030002, and by the National Natural Science Foundation of China under grant Nos. 31570364 and 61871293.

\section{Conflict of Interest}

The authors declare no conflict of interest.

\section{References}

1. SHENG G.P., YU H.Q., LI X.Y. Extracellular polymeric substances (EPS) of microbial aggregates in biological wastewater treatment systems: a review. Biotechnol. Adv. 28, 882, 2010.

2. WANG Z.P., LIU L.L., YAO J., CAI W.M. Effects of extracellular polymeric substances on aerobic granulation in sequencing batch reactors. Chemosphere. 63, 1728, 2006.

3. GRETCHEN R.B., STEPHEN M. B., ALEJANDRO G., JULIE Z., TAMMY L., JOSH E. Feeding dynamics of the copepod Diacyclops thomasi before, during and following filamentous cyanobacteria blooms in a large, shallow temperate lake. Hydrobiologia. 705 (1), 101, 2013.

4. MA W.X., HUANG T.L., LI X. Study of the application of the water-lifting aerators to improve the water quality of a stratified, eutrophicated reservoir. Ecol. Eng. 83, 281, 2015.

5. YAN L., LIU Y., WEN Y., REN Y., HAO G., ZHANG, Y. Role and significance of extracellular polymeric substances from granular sludge for simultaneous removal of organic matter and ammonia nitrogen. Bioresour. Technol. 179, 460, 2014.

6. HUANG J., GRAHAM N., TEMPLETON M. R., ZHANG Y., COLLINS C., NIEUWENHUIJSEN, M. A comparison of the role of two blue-green algae in THM and HAA formation. Water Res. 43, 3009, 2009.

7. QU F.S., LIANG H., HE J.G., MA J., WANG Z.Z., YU H., LI G.B. Characterization of dissolved extracellular organic matter (dEOM) and bound extracellular organic matter (bEOM) of microcystis aeruginosa and their 
impacts on UF membrane fouling. Water Res. 46, 2881, 2012.

8. DE PHILIPPIS R., VINCENZINI M. Exocellular polysaccharides from cyanobacteria and their possible applications. FEMS Microbiol Rev. 22, 151, 2006.

9. GAN N.Q., XIAO Y., ZHU L., WU Z.X., LIU J., HU C.L., SONG L.R. The role of microcystins in maintaining colonies of bloom-forming microcystis spp. Environ. Microbiol. 14, 730, 2012.

10. KLOCK J.H., WIELAND A., SEIFERT R., MICHAELIS W. Extracellular polymeric substances (EPS) from cyanobacterial mats: characterisation and isolation method optimisation. Mar. Biol. 152, 1077, 2007.

11. PEREIRA S., ZILLE A., MICHELETTI E., MORADASFERREIRA P., DE PHILIPPIS R., TAMAGNINI P. Complexity of cyanobacterial exopolysaccharides: composition, structures, inducing factors and putative genes involved in their biosynthesis and assembly. FEMS Microbiol. Rev. 33, 917, 2009.

12. XU H., CAI H., YU G., JIANG H. Insights into extracellular polymeric substances of cyanobacterium Microcystis aeruginosa using fractionation procedure and parallel factor analysis. Water Res. 47, 2005, 2013

13. XU H., JIANG H., YU G., YANG L. Towards understanding the role of extracellular polymeric substances in cyanobacterial Microcystis aggregation and mucilaginous bloom formation. Chemosphere, 117, 815, 2014.

14. PIONTEK M., CZYŻEWSKA W., MANKIEWICZBOCZEK J. The Occurence of Cyanobacteria Blooms in the Obrzyca River Catchment Area (Poland), a Source of Drinking Water. Pol. J. Environ. Stud. 26 (3), 1191, 2017.

15. SARNELLE O., WHITE J.D., HORST G.P., HAMILTON S.K. Phosphorus addition reverses the positive effect of zebra mussels (Dreissena polymorpha) on the toxic cyanobacterium, Microcystis aeruginosa. Water Res. 46, 3471, 2012.

16. PAJDAK-STOS A., FIAŁKOWSKA E., FYDA. J. Phormidium autumnale (Cyanobacteria) defense against three ciliate grazer species. Aquat. Microb. Ecol. 23, 237, 2001.
17. HENDERSON R.K., BAKER A., PARSONS S.A., JEFFERSON B. Characterisation of algogenic organic matter extracted from cyanobacteria, green algae and diatoms. Water Res. 42, 3435, 2008.

18. LI L., GAO N., DENG Y., YAO J., ZHANG K. Characterization of intracellular \& extracellular algae organic matters (AOM) of Microcystic aeruginosa and formation of AOM-associated disinfection byproducts and odor \& taste compounds. Water Res. 46, 1233, 2012.

19. REN L.Y., HONG Z.N., QIAN W. Adsorption mechanism of extracellular polymeric substances from two bacteria on Ultisol and Alfisol. Environ Pollut. 39, 2018.

20. LIU H., FANG H.H. Extraction of extracellular polymeric substances (EPS) of sludges. J. Biotechnol. 95, 249, 2002.

21. CHI Z., SU C.D., LU W.D. A new exopolysaccharide produced by marine Cyanothece sp. 113. Bioresour. Technol. 98, 1329, 2007.

22. XIAO W., ZHANG Y., GAO M., HUANG X., ZHONG Q., WANG W., GU K. Isolation and cultivation of a wild microcytin-RR-producing cyanobacterium and verification of its toxin by high performance liquid chromatography and acute oral toxicity. Chin. J. Prevent. Med. 48 (7), 622, 2014.

23. WANG L., WANG X.Y., JIN X.B., XU J.P., ZHANG H.Y., YU J.B., SUN Q., GAO C.,WANG L.B. Analysis of algae growth mechanism and water bloom prediction under the effect of multi-affecting factor. Saudi. J. Biol. Sci. 24 (3), $556,2017$.

24. GHAFFAR S., STEVENSON R.J., KHAN Z. Effect of phosphorus stress on Microcystis aeruginosa growth and phosphorus uptake. PLOS ONE. 2017, 2017.

25. CHEN J., TOPTYGIN D., BRAND L., KING J. Mechanism of the efficient tryptophan fluorescence Quenching in Human gammaD-Crystallin studied by Time-Resolved Fluorescence. Biochemistry, 47, 10705, 2008.

26. SORRELS C.M., PROTEAU P.J., GERWICK W.H. Organization, evolution, and expression analysis of the biosynthetic gene cluster for scytonemin, a cyanobacterial UV-absorbing pigment. J. Appl. Environ. Microbiol. 75, 4861, 2009. 